\title{
Early Injection Laryngoplasty With a Long-Lasting Material in Patients With Potentially Recoverable Unilateral Vocal Fold Paralysis
}

\author{
Nayeon Choi (i) $\cdot$ HoKyung Jin (10) Hack Jung Kim (iD $\cdot$ Young-Ik Son (iD \\ Department of Otorhinolaryngology-Head and Neck Surgery, Samsung Medical Center, \\ Sungkyunkwan University School of Medicine, Seoul, Korea
}

Objectives. Injection laryngoplasty (IL) is one of the major options for treatment of unilateral vocal fold paralysis (UVFP). Early IL in patients with abrupt-onset UVFP can reduce hoarseness and aspiration-related discomfort and complications. Temporary or short-lasting materials are recommended for the early IL since permanent or long-lasting materials may negatively affect the voice quality when vocal fold motion is spontaneously recovered.

Methods. Patients who received IL with a long-lasting material (ArteSense) within 1 month following development of postoperative UVFP were enrolled for this study. They were categorized into a spontaneously recovered group $(\mathrm{n}=30)$ and unrecovered group $(n=276)$ from UVFP. The subjective and objective voice parameters were collected before and 6 months after IL. Patients' demographics and collected voice parameters were compared between the two groups.

Results. Age and sex distributions were not different between the two groups. Aspiration symptom, maximum phonation time (MPT), jitter, shimmer, noise to harmonic ratio (NHR), overall grade (G) and breathiness (B) of GRBAS (grade of dysphonia, roughness, breathiness, asthenia, strain) scale score, and voice handicap index (VHI) were significantly improved after IL in both groups. The amounts of improvement in MPT, jitter, shimmer, NHR and VHI were significantly greater in the recovered group. There was no IL-related complication including granuloma formation, hypersensitivity to injection material, or worsening of hoarseness.

Conclusion. Early IL with a long-lasting material can be safely performed to improve voice quality and to reduce aspiration episode for the patients with postoperative UVFP, regardless of recovery from the paralysis. The amounts of improvement in the subjective and objective voice parameters were significantly greater in patients who showed spontaneous recovery from UVFP when compared with those in patients who did not.

Keywords. Vocal Cords; Paralysis; Laryngoplasty; Early Intervention; Injection

\section{INTRODUCTION}

Unexpected unilateral vocal fold paralysis (UVFP) is one of the embarrassing complications of the thoracic and neck surgery.

\footnotetext{
- Received March 21, 2019

Revised June 10, 2019

Accepted June 26, 2019

- Corresponding author: Young-Ik Son

Department of Otorhinolaryngology-Head and Neck Surgery, Samsung

Medical Center, Sungkyunkwan University School of Medicine,

81 Irwon-ro, Gangnam-gu, Seoul 06351, Korea

Tel: +82-2-3410-3579, Fax: +82-2-3410-6987

E-mail: yison@skku.edu
}

Clinical courses of postoperative UVFP are diverse including wide range of spontaneous recovery, synkinesis and permanent paralysis. Most of the spontaneous recovery from UVFP is obtained within a year, but it is difficult to predict whether UVFP will recover and when it will recover [1].

Abrupt-onset UVFP may cause hoarseness and aspiration, which can result in poor quality of life, longer hospital stay, and delay in the planned treatments [2-4]. Patients with abrupt-onset UVFP have higher chances of invasive procedures including bronchoscopy and tracheostomy [4]. Injection laryngoplasty (IL) became a major treatment option for the UVFP because of its noninvasiveness and development of biocompatible injection

Copyright (C) 2019 by Korean Society of Otorhinolaryngology-Head and Neck Surgery.

This is an open-access article distributed under the terms of the Creative Commons Attribution Non-Commercial License (http://creativecommons.org/licenses/by-nc/4.0)

which permits unrestricted non-commercial use, distribution, and reproduction in any medium, provided the original work is properly cited. 
materials [3]. Recent studies have supported for the early IL in case of postoperative UVFP, since it can be easily performed without adding further morbidities and it will help to prevent or reduce UVFP-related discomfort and complications [5,6].

When performing an early IL in patients with potentially recoverable UVFP, temporary or short-lasting injection materials are preferred since long-acting materials may adversely affect the voice quality when UVFP is spontaneously recovered. Short-lasting materials including hyaluronic acid, collagen, or cellulose are usually resolved within 3 to 6 months [3]. Regarding the early IL for postoperative UVFP, most of the previous studies used short-lasting materials, therefore, many patients required additional procedures $[7,8]$. There is a study which used a long-lasting material for the early IL in potentially recoverable UVFP patients; it reported that the patients achieved adequate voice quality without further needs of open phonosurgery, but it did not provide objective voice quality data [9].

In this study, we evaluated the voice outcomes of early IL with a long-lasting material in patients with potentially recoverable postoperative UVFP and compared the subjective and objective voice parameters between the recovered and unrecovered patients from UVFP.

\section{MATERIALS AND METHODS}

\section{Patients}

This retrospective study was approved by Samsung Medical Center Institutional Review Board (IRB No. 2018-08-062) and informed consent was exempted. The medical records of patients who received IL between May 2004 and May 2016 at the authors' institution were reviewed. Enrolled patients received an IL within a month after onset of UVFP following surgeries of the thyroid, esophagus, thymus or lung cancers. The patients who had no possibility of spontaneous vocal fold motion recovery due to the transection of the vagus nerve, recurrent laryngeal nerve or direct tumor invasion to these nerves were excluded.

Total 306 patients were enrolled, and they were classified into two groups; spontaneously recovered from UVFP (recovered

\section{HII G H L I G H T S}

- Early injection laryngoplasty (IL) with a long-lasting material can be safely performed in patients with postoperative unilateral vocal fold paralysis (UVFP).

- Subjective and objective voice parameters significantly improved after early IL, regardless of recovery status from the paralysis.

- The amounts of improvement in the subjective and objective voice parameters were significantly greater in patients who showed spontaneous recovery from UVFP. group, $n=30)$ and unrecovered group $(n=276)$. Vocal fold mobility and subjective and objective voice parameters were evaluated at 6 months after IL procedures. Most of the patients were evaluated again at 1 year after IL to assess delayed complications including granuloma formation and to determine the need for further management of UVFP.

\section{IL procedure}

All of IL procedures were performed through cricothyroid membrane approach under local anesthesia. For local anesthesia, patients inhaled $4 \%$ lidocaine using nebulizer for 5 minutes, and $2 \%$ lidocaine injection was performed at the subcutaneous layer around the cricothyroid membrane. Flexible fiberoptic nasopharyngoscope (VNL-1530T; Pentax, Tokyo, Japan) was introduced through nasal cavity to visualize the vocal fold. The cricothyroid membrane was identified by palpation and 23-guage spinal needle was inserted into paralyzed vocal fold until the needle tip was bluntly seen at the lateral and anterior side of the vocal process of arytenoid cartilage. We used ArteSense (European Medical Contract Manufacturing B.V., Nijmegen, The Netherlands) which consists of $20 \%$ volume of polymethylmethacrylate (PMMA) microspheres suspended in $80 \%$ volume of denatured bovine collagen. In average, $0.7 \mathrm{~mL}$ of ArteSense was injected to get slight overcorrection of volume of the paralyzed vocal fold. Cautions were given to inject slowly, to keep the plane at deeper layer of vocal ligament and subglottic portion of the paralyzed vocal fold. Patients were observed for 2 hours after IL to check acute complications including allergic reaction, bleeding and dyspnea.

\section{Voice evaluations}

The objective and subjective voice evaluations were performed before and 6 months after IL. Subjective aspiration symptom was also checked. Laryngeal examination using stroboscope (Pentax, Lincoln Park, NJ, USA) was performed to evaluate the vocal fold mobility, patterns of glottic closure and vibratory phase symmetry. Maximum phonation time (MPT), jitter percentage, shimmer percentage, and noise to harmonic ratio (NHR) were evaluated for aerodynamic and acoustic analyses. These parameters were calculated using the software of Computerized Speech Lab (Kay Elemetrics, Pine Brook, NJ, USA). The GRBAS (grade of dysphonia, roughness, breathiness, asthenia, strain) scales [10] were used for perceptual evaluation by two experienced speech language pathologists. The patients replied Korean version of the voice handicap index (VHI)-30 and VHI-10 which is a patients-based self-rating questionnaires including the subscales of the functional, physical, and emotional aspects of the voice impairment [11,12].

\section{Statistical analysis}

Baseline characteristics and voice related clinical factors were compared between the recovered and unrecovered group. Voice 
parameters obtained before and after IL were compared by paired sample $t$-test in each group. The amounts of improvement in voice parameters ( $\Delta$ voice parameter) after IL were compared between the two groups by the Mann-Whitney $U$-test. A $P$-value $<0.05$ was considered as statistically significant. Statistical analyses were performed using IBM SPSS ver. 20.0 (IBM Corp., Armonk, NY, USA).

\section{RESULTS}

\section{Baseline characteristics of the enrolled patients}

Baseline characteristics of the patients were shown at Table 1. The age at the time of IL and sex distribution were not different between the two groups. The etiologies of vocal fold paralysis were neck surgery $(n=12,40.0 \%)$ and thoracic surgery $(n=18$,

Table 1. Baseline characteristics of the spontaneously recovered group and unrecovered group from unilateral vocal fold paralysis

\begin{tabular}{lccc}
\hline Clinical factor & $\begin{array}{c}\text { Recovered } \\
(\mathrm{n}=30)\end{array}$ & $\begin{array}{c}\text { Unrecovered } \\
(\mathrm{n}=276)\end{array}$ & $P$-value \\
\hline Baseline characteristics & & & \\
Age (yr) & $53.4 \pm 11.7$ & $55.3 \pm 14.2$ & 0.286 \\
Sex (male:female) & $15: 15$ & $153: 123$ & 0.570 \\
& $(50.0: 50.0)$ & $(55.4: 44.6)$ & \\
Cause of procedure & & & 0.281 \\
$\quad$ Neck surgery & $12(40.0)$ & $139(50.4)$ & \\
$\quad$ Thoracic \& esophagus surgery & $18(60.0)$ & $137(49.6)$ & \\
Vocal fold recovery state & & & \\
$\quad$ Full recovery & $23(76.7)$ & & \\
$\quad$ Partial recovery & $7(23.3)$ & & \\
\hline
\end{tabular}

Values are presented as mean \pm standard deviation or number (\%).
$60.0 \%)$ in the recovered group, and neck surgery $(n=139,50.4 \%)$ and thoracic surgery $(n=137,49.6 \%)$ in the unrecovered group, which were not statistically different between the two groups $(P=0.281)$.

\section{Voice evaluations before and after IL}

The values of subjective and objective voice parameters were summarized in Table 2 . In the recovered group, nine patients (30\%) reported aspiration symptoms before IL, which improved in all patients after IL. In contrast, in the unrecovered group, aspiration symptom was observed in 54 patients $(19.6 \%)$ before IL and seven patients (2.5\%) after IL. The MPT significantly improved after IL in both groups $(P<0.001)$. The MPT improved from $4.9 \pm 2.9$ to $12.2 \pm 5.8$ seconds in the recovered group and from $5.0 \pm 3.9$ to $7.7 \pm 5.5$ seconds in the unrecovered group. The jitter, shimmer and NHR also significantly improved in both groups $(P<0.001)$. The jitter and shimmer more markedly improved in the recovered group than in the unrecovered group. Regarding GRBAS scores, overall grade of dysphonia $(G)$, breathiness (B) and asthenia (A) improved significantly in both groups $(P<0.001)$. The VHI-30 and VHI-10 improved significantly after IL in both groups $(P<0.001)$.

Changes in voice parameters of each patient of the recovered group are depicted in Fig. 1. Most of the patients showed improvement of jitter, shimmer, NHR, MPT, VHI-30 and G. None of the recovered group patients experienced worsening of VHI30 and $\mathrm{G}$ by the early IL with a long-lasting material. Stroboscopic evaluations confirmed that mucosal waves were not hampered in all of the patients. Follow-up laryngoscopic evaluation at 1 year after IL revealed no case of delayed complications including granuloma formation.

Table 2. Voice outcomes before and after early injection laryngoplasty in patients with spontaneously recovered and unrecovered unilateral vocal fold paralysis

\begin{tabular}{|c|c|c|c|c|c|c|}
\hline \multirow{2}{*}{ Variable } & \multicolumn{3}{|c|}{ Recovered $(n=30)$} & \multicolumn{3}{|c|}{ Unrecovered $(n=276)$} \\
\hline & Before & After & $P$-value & Before & After & $P$-value \\
\hline Aspiration & $9(30)$ & 0 & $<0.001$ & $54(19.6)$ & $7(2.5)$ & $<0.001$ \\
\hline MPT (sec) & $4.9 \pm 2.9$ & $12.2 \pm 5.8$ & $<0.001$ & $5.0 \pm 3.9$ & $7.7 \pm 5.5$ & $<0.001$ \\
\hline Jitter (\%) & $5.5 \pm 4.3$ & $1.7 \pm 1.6$ & $<0.001$ & $5.8 \pm 5.2$ & $3.4 \pm 3.9$ & $<0.001$ \\
\hline Shimmer (\%) & $11.3 \pm 8.4$ & $4.0 \pm 3.2$ & $<0.001$ & $10.9 \pm 7.7$ & $7.5 \pm 6.5$ & $<0.001$ \\
\hline $\mathrm{NHR}$ & $0.2 \pm 0.2$ & $0.1 \pm 0.0$ & $<0.001$ & $0.3 \pm 0.2$ & $0.2 \pm 0.2$ & $<0.001$ \\
\hline \multicolumn{7}{|l|}{ GRBAS score } \\
\hline Grade of dysphonia & $2.7 \pm 0.5$ & $0.7 \pm 0.7$ & $<0.001$ & $2.5 \pm 0.7$ & $1.3 \pm 0.9$ & $<0.001$ \\
\hline Roughness & $0.3 \pm 0.8$ & $0.4 \pm 0.6$ & 0.424 & $0.5 \pm 0.9$ & $0.4 \pm 0.7$ & 0.272 \\
\hline Breathiness & $2.7 \pm 0.5$ & $0.5 \pm 0.7$ & $<0.001$ & $2.4 \pm 0.7$ & $1.2 \pm 0.9$ & $<0.001$ \\
\hline Asthenia & $0.1 \pm 0.5$ & 0 & $<0.001$ & $0.3 \pm 0.7$ & $0.1 \pm 0.4$ & $<0.001$ \\
\hline Strain & 0 & $0.0 \pm 0.2$ & 0.500 & $0.1 \pm 0.3$ & $0.1 \pm 0.3$ & 0.424 \\
\hline VHI-30 & $82.5 \pm 28.8$ & $27.7 \pm 29.0$ & $<0.001$ & $79.5 \pm 26.1$ & $55.8 \pm 31.0$ & $<0.001$ \\
\hline $\mathrm{VHI}-10$ & $29.0 \pm 8.3$ & $9.4 \pm 10.3$ & $<0.001$ & $28.6 \pm 8.7$ & $20.3 \pm 11.6$ & $<0.001$ \\
\hline
\end{tabular}

Values are presented as number (\%) or mean \pm standard deviation.

MPT, maximum phonation time; NHR, noise to harmonic ratio; GRBAS, grade of dysphonia, roughness, breathiness, asthenia, strain; VHI, voice handicap index. 

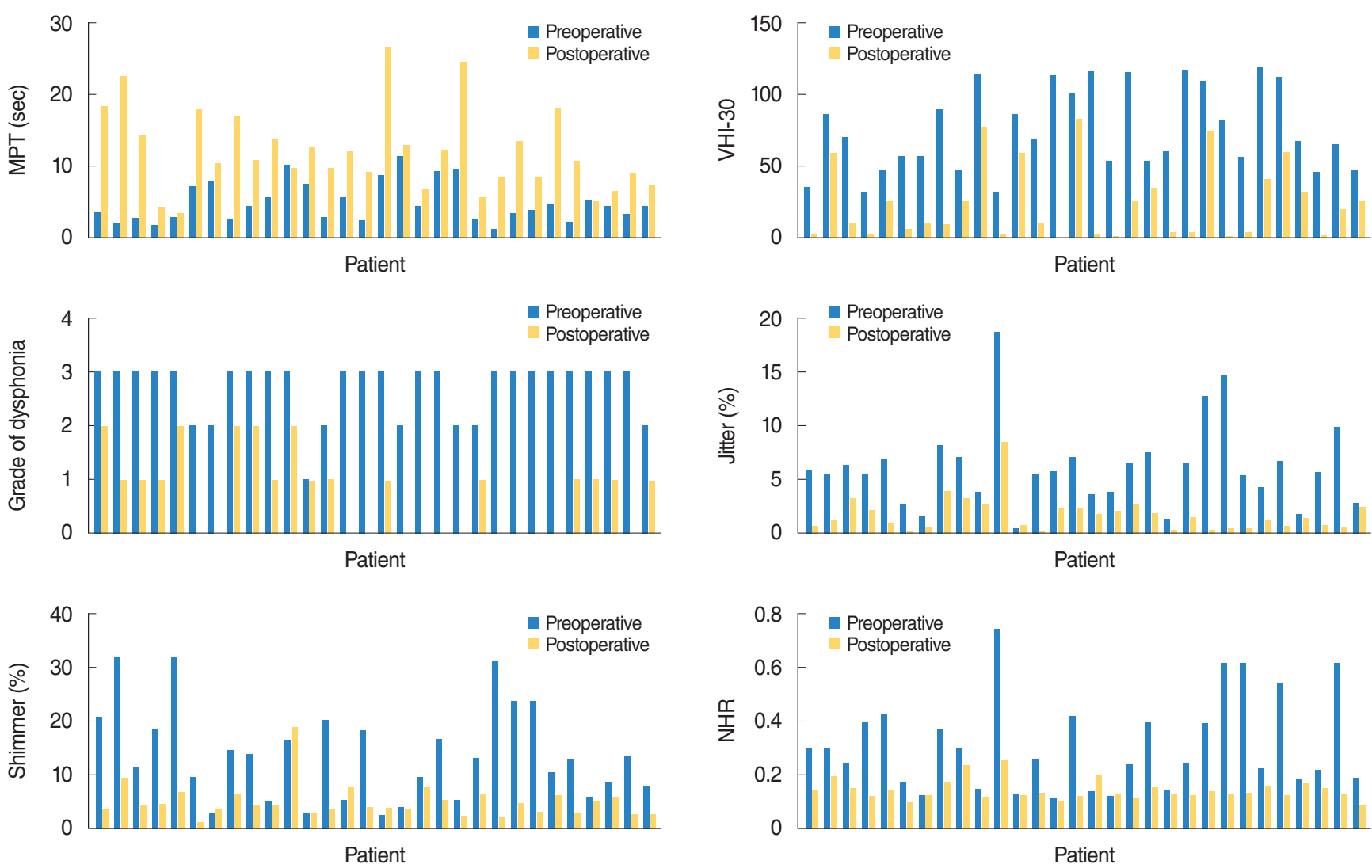

Fig. 1. Voice parameters before and after injection laryngoplasty in each patient $(n=30)$ of spontaneous recovery from unilateral vocal fold paralysis. MPT, maximum phonation time; VHI, voice handicap index; NHR, noise to harmonic ratio.

\section{Amounts of voice improvement between the recovered and unrecovered group}

The amounts of voice improvement were summarized in Table 3. $\Delta \operatorname{MPT}(\Delta$, difference between before and after injection laryngoplasty) was significantly longer $(P<0.001)$ in the recovered group (7.3 \pm 6.9 seconds) than in the unrecovered group $(2.7 \pm$ 5.6 seconds). $\Delta$ Jitter was significantly greater $(P=0.026)$ in the recovered group $(-3.8 \% \pm 3.8 \%)$ than in the unrecovered group $(-2.3 \% \pm 5.2 \%)$. In contrast, $\triangle$ Shimmer and $\triangle$ NHR were not different between the two groups $(P=0.172$ and $P=0.315$, respectively). Regarding the GRBAS scales, $G$ and $B$ improved more in the recovered group $(P<0.001) . \Delta \mathrm{VHI}-30$ and $\Delta \mathrm{VHI}-10$ showed significantly greater improvement $(P<0.001)$ in the recovered group than in the unrecovered group.

\section{DISCUSSION}

Postoperative UVFP is not uncommon complication of the extensive neck or thoracic surgery. The prognosis of UVFP is difficult to predict. Laryngeal electromyography is a good predictor of poor prognosis for UVFP, but accuracy of prediction for complete recovery is only $12.8 \%[13,14]$. Because it is difficult to
Table 3. Variance of voice parameters between before and after injection laryngoplasty in patients with spontaneously recovered and unrecovered unilateral vocal fold paralysis

\begin{tabular}{lccr}
\hline Variable & $\begin{array}{c}\text { Recovered } \\
(\mathrm{n}=30)\end{array}$ & $\begin{array}{c}\text { Unrecovered } \\
(\mathrm{n}=276)\end{array}$ & $P$-value \\
\hline$\triangle \mathrm{MPT}(\mathrm{sec})$ & $7.3 \pm 6.9$ & $2.7 \pm 5.6$ & $<0.001$ \\
$\Delta$ Jitter (\%) & $-3.8 \pm 3.8$ & $-2.3 \pm 5.2$ & 0.026 \\
$\Delta$ Shimmer (\%) & $-6.1 \pm 8.8$ & $-3.4 \pm 8.5$ & 0.172 \\
$\Delta \mathrm{NHR}$ & $-0.1 \pm 0.2$ & $-0.1 \pm 0.4$ & 0.315 \\
$\Delta$ GRBAS score & & & \\
$\quad$ Grade of dysphonia & $-2.0 \pm 0.9$ & $-1.1 \pm 1.0$ & $<0.001$ \\
$\quad$ Roughness & $0.1 \pm 1.0$ & $-0.1 \pm 0.9$ & 0.175 \\
$\quad$ Breathiness & $-2.2 \pm 0.8$ & $-1.2 \pm 1.0$ & $<0.001$ \\
$\quad$ Asthenia & $-0.1 \pm 0.5$ & $-0.2 \pm 0.7$ & 0.509 \\
$\quad$ Strain & $0.0 \pm 0.2$ & $-0.0 \pm 0.4$ & 0.341 \\
$\Delta$ VHI30 & $-54.9 \pm 31.5$ & $-23.7 \pm 30.9$ & $<0.001$ \\
$\Delta$ VHI10 & $-19.6 \pm 9.9$ & $-7.7 \pm 11.3$ & $<0.001$ \\
\hline
\end{tabular}

Values are presented as mean \pm standard deviation.

$\Delta$, difference between before and after injection laryngoplasty; MPT, maximum phonation time; NHR, noise to harmonic ratio; GRBAS, grade of dysphonia, roughness, breathiness, asthenia, strain; $\mathrm{VHI}$, voice handicap index.

clearly foretell whether and when UVFP will recover, management of postoperative UVFP should be focused to reduce the 
patients' discomfort and related complications. IL, medialization thyroplasty, and arytenoid adduction are available surgical options for the management of UVFP $[15,16]$. Early IL has become a major treatment option for the acute-onset postoperative UVFP because of its effectiveness, noninvasiveness and low rate of complications $[5,6]$. The best timing for early $\mathrm{IL}$ is controversial. Previous studies defined an early injection as from 1 day to 6 months after occurrence of UVFP [5-7]. In our institution, we usually performed IL within 1 month in the hope to provide the better quality of life and to avoid a delay in the planned treatment for their main diseases.

Injection materials can be categorized into temporary (shortlasting) and permanent (long-lasting) materials according to the duration of effects in the body. Permanent (long-lasting) injection materials include autologous fat, calcium hydroxylapatite (Radiesse), PMMA (ArteSense) and polydimethylsiloxane (or particulate silicone). Temporary injection materials include bovine gelatin (Gelfoam, Surgifoam), collagen-based products (Cymetra, Zyplast, Cosmoplast/Cosmoderm), hyaluronic acid (Restylane, Hylaform), and carboxymethylcellulose (Radiesse Voice Gel). According to the literatures, "permanent/long-lasting" materials typically refer to lasting more than 1 year $[17,18]$. In 2006, ArteFill (the same product of ArteSense) became the first permanent injectable wrinkle filler to receive U.S. Food and Drug Administration approval [19]. ArteSense is a mixture of denatured bovine collagen (short-lasting) and PMMA (long-lasting component). The PMMA microspheres become encapsulated with the collagen fibers secreted by the patients' own fibroblasts and stabilized for more than several years [20]. A 5 -year followup study in U.S. has shown the same wrinkle improvement as seen at 6 months. Long-term follow-up in European patients has shown wrinkle correction lasting up to 15 years [19].

In patients with potentially recoverable UVFP, IL with a temporary or short-lasting material is usually preferred because there are concerns that a permanent or long-lasting material may disturb the natural course of vocal fold recovery and/or injection material remained in vocal fold may reduce vocal fold mucosal wave, which can result in unfavorable voice quality. However, even collagen, a representative of short-lasting materials not infrequently have long term effects on voice quality [21]. According to previous reports, satisfactory voice outcomes were maintained after IL with a long-lasting material in patients who later showed spontaneous recovery from UVFP $[9,22]$. In addition, IL with a long-lasting material is a useful procedure not only for UVFP but also for the other types of glottal insufficiency including vocal atrophy, in which bilateral vocal folds are fully mobile. It means that IL with a long-lasting material will be beneficial in selected cases of glottal insufficiency regardless of vocal fold mobility. A meta-analysis reported that complete recovery occurred in $13 \%-83 \%$ and partial recovery occurred in $19 \%-83 \%$ of UVFP [1]. Partially recovered vocal folds often result in glottal insufficiency because of the atrophy of intrinsic vocal fold muscles as well as incomplete adduction during phonation. Therefore, IL will be helpful for the more complete glottal closure of these partially recovered vocal folds.

In this study, none of the patients experienced acute adverse reactions including dyspnea, bleeding, hematoma, or allergic reaction to the injection materials. Theoretically bovine collagen allergy testing is required to minimize the risk of hypersensitivity reactions. The substance used as a carrier for the microbeads of ArteSense is the collagen derivative, atelocollagen. Enzymatic digestion of the antigenic end of natural bovine collagen minimizes the risk of allergic reactions (product inserts). Reports of allergic reactions to ArteFill are extremely rare (0.2\%) [19]. PMMA microsphere of ArteSense has a smooth, round surface and is suspended in collagen; it allows tissue ingrowth into the interstitial space around the microspheres. This decreases the displacement of the filler. PMMA microsphere is big enough (30 to $50 \mu \mathrm{m}$ in diameter) to avoid phagocytosis by macrophages and giant cell formation resulting in inflammation [23]. During the last 15 years, the authors have used ArteSense (formerly labeled as Artecoll) for more than 1,500 cases of IL. There has been no long-term or delayed complication including migration, granuloma or foreign body reaction. Mucosal waves of the vocal fold did not decrease regardless of spontaneous recovery from UVFP.

A previous study of potentially recoverable UVFP investigated voice related quality of life but not the other objective voice parameters [9]. According to a meta-analysis study, MPT, GRBAS, and mean airflow rate were recognized as significant indicators of voice quality in patients with UVFP [24]. In this study, MPT, jitter, shimmer, NHR, GRBAS, and VHI significantly improved after early IL with a long-lasting material in both groups. The patients of recovered group showed significantly greater improvement in MPT, jitter, and VHI than unrecovered patients. Notably, none of the recovered patients reported worsening of VHI-30 or G in GRBAS. Based on these results, we support that early IL with a long-lasting material is effective in improving voice quality even in patients who may show spontaneous recovery from postoperative UVFP.

There are several limitations of this study. Comparing voice outcomes between the recovered and unrecovered group does not necessarily tell us the true effects of early IL with a longlasting material, especially for the patients of recovered group. In the recovered group, their voice outcomes will be affected by both IL procedure and natural course of recovery from UVFP. To specify the effects of IL in the recovered group, data should be compared in patients between with and without IL procedure. Moreover, we used only a long-lasting injection material and performed IL within a month of UVFP onset. Further studies comparing short-lasting with long-lasting materials and early with delayed timing of IL will provide more reliable conclusions regarding the use of a long-lasting material for the early $\mathrm{IL}$ in potentially recoverable UVFP patients. In addition, we analyzed 
only short-term follow-up data (6 months). If evaluated at more than 1 year of UVFP onset, more patients in the unrecovered group may be re-classified into the recovered group and voice outcomes may be different according to the degree of recovery from UVFP. Moreover, long-term follow-up will provide us more information regarding advantages as well as disadvantages of using long-lasting material for the early IL in patients with acute-onset UVFP.

When patients have significant discomfort in their voice or swallowing because of abrupt-onset UVFP after the neck or thoracic surgery, early IL with a long-lasting material can be safely performed to improve their voice quality and to reduce aspiration-related discomfort, even in patients who will show a spontaneous recovery from UVFP.

\section{CONFLICT OF INTEREST}

No potential conflict of interest relevant to this article was reported.

\section{ORCID}

Nayeon Choi https://orcid.org/0000-0002-0962-0594

HoKyung Jin https://orcid.org/0000-0002-7278-0320

Hack Jung Kim https://orcid.org/0000-0002-1294-9315

Young-lk Son https://orcid.org/0000-0002-2114-8085

\section{AUTHOR CONTRIBUTIONS}

Conceptualization: NC, YIS. Data curation \& Formal analysis: all authors. Methodology \& Project administration: NC, YIS. Visualization \& Writing - original draft: all authors. Writing - review \& editing: NC, YIS.

\section{REFERENCES}

1. Sulica L. The natural history of idiopathic unilateral vocal fold paralysis: evidence and problems. Laryngoscope. 2008 Jul;118(7):1303-7.

2. Rosenthal LH, Benninger MS, Deeb RH. Vocal fold immobility: a longitudinal analysis of etiology over 20 years. Laryngoscope. 2007 Oct;117(10):1864-70.

3. Kwon TK, Buckmire R. Injection laryngoplasty for management of unilateral vocal fold paralysis. Curr Opin Otolaryngol Head Neck Surg. 2004 Dec;12(6):538-42.

4. DiLisio RP, Mazzeffi MA, Bodian CA, Fischer GW.Vocal cord paralysis after aortic surgery. J Cardiothorac Vasc Anesth. 2013 Jun;27(3): 522-7.

5. Friedman AD, Burns JA, Heaton JT, Zeitels SM. Early versus late injection medialization for unilateral vocal cord paralysis. Laryngo- scope. 2010 Oct;120(10):2042-6.

6. Jang JY, Lee G, Ahn J, Son YI. Early voice rehabilitation with injection laryngoplasty in patients with unilateral vocal cord palsy after thyroidectomy. Eur Arch Otorhinolaryngol. 2015 Dec;272(12): 3745-50.

7. Graboyes EM, Bradley JP, Meyers BF, Nussenbaum B. Efficacy and safety of acute injection laryngoplasty for vocal cord paralysis following thoracic surgery. Laryngoscope. 2011 Nov;121(11):2406-10.

8. Tan M,Woo P. Injection laryngoplasty with micronized dermis: a 10year experience with 381 injections in 344 patients. Laryngoscope. 2010 Dec;120(12):2460-6.

9. Arviso LC, Johns MM 3rd, Mathison CC, Klein AM. Long-term outcomes of injection laryngoplasty in patients with potentially recoverable vocal fold paralysis. Laryngoscope. 2010 Nov;120(11):223740.

10. Isshiki N, Okamura H, Tanabe M, Morimoto M. Differential diagnosis of hoarseness. Folia Phoniatr (Basel). 1969;21(1):9-19.

11. Rosen CA, Murry T, Zinn A, Zullo T, Sonbolian M. Voice handicap index change following treatment of voice disorders. J Voice. 2000 Dec;14(4):619-23.

12. Rosen CA, Lee AS, Osborne J, Zullo T, Murry T. Development and validation of the voice handicap index-10. Laryngoscope. 2004 Sep; 114(9):1549-56.

13. Sittel C, Stennert E, Thumfart WF, Dapunt U, Eckel HE. Prognostic value of laryngeal electromyography in vocal fold paralysis. Arch Otolaryngol Head Neck Surg. 2001 Feb;127(2):155-60.

14. Rickert SM, Childs LF, Carey BT, Murry T, Sulica L. Laryngeal electromyography for prognosis of vocal fold palsy: a meta-analysis. Laryngoscope. 2012 Jan;122(1):158-61.

15. Siu J,Tam S, Fung K.A comparison of outcomes in interventions for unilateral vocal fold paralysis: a systematic review. Laryngoscope. 2016 Jul;126(7):1616-24.

16. Vij S, Gupta AK, Vir D. Voice quality following unilateral vocal fold paralysis: a randomized comparison of therapeutic modalities. J Voice. 2017 Nov;31(6):774.

17. Salinas JB, Chhetri DK. Injection laryngoplasty: techniques and choices of fillers. Curr Otorhinolaryngol Rep. 2014 Jun;2(2):131-6.

18. Mallur PS, Rosen CA. Vocal fold injection: review of indications, techniques, and materials for augmentation. Clin Exp Otorhinolaryngol. 2010 Dec;3(4):177-82.

19. Lemperle G, Knapp TR, Sadick NS, Lemperle SM. ArteFill permanent injectable for soft tissue augmentation: I. mechanism of action and injection techniques. Aesthetic Plast Surg. 2010 Jun;34(3):26472.

20. Cohen SR, Berner CF, Busso M, Gleason MC, Hamilton D, Holmes RE, et al.ArteFill: a long-lasting injectable wrinkle filler material: summary of the U.S. Food and Drug Administration trials and a progress report on 4- to 5-year outcomes. Plast Reconstr Surg. 2006 Sep;118 (3 Suppl):64S-76S.

21. Pearl AW, Woo P, Ostrowski R, Mojica J, Mandell DL, Costantino P. A preliminary report on micronized AlloDerm injection laryngoplasty. Laryngoscope. 2002 Jun;112(6):990-6.

22. Min JY, Hong SD, Kim K, Son YI. Long-term results of Artecoll injection laryngoplasty for patients with unilateral vocal fold motion impairment: safety and clinical efficacy.Arch Otolaryngol Head Neck Surg. 2008 May;134(5):490-6.

23. Attenello NH, Maas CS. Injectable fillers: review of material and properties. Facial Plast Surg. 2015 Feb;31(1):29-34.

24. Desuter G, Dedry M, Schaar B, van Lith-Bijl J, van Benthem PP, Sjogren EV.Voice outcome indicators for unilateral vocal fold paralysis surgery: a review of the literature. Eur Arch Otorhinolaryngol. 2018 Feb;275(2):459-68. 